University of Nebraska - Lincoln

DigitalCommons@University of Nebraska - Lincoln

2005

\title{
Molecular Epidemiology of Plasmodium falciparum Resistance to Antimalarial Drugs in Indonesia
}

Din Syafruddin

Eijkman Institute for Molecular Biology, din@eijkman.go.id

Puji B. S. Asih

Eijkman Institute for Molecular Biology

Gerard J. Casey

The Walter and Eliza Hall Institute of Medical Research

Jason Maguire

United States Naval Medical Research Unit \# 2

J. Kevin Baird

United States Naval Medical Research Unit \# 2, jkevinbaird@yahoo.com

See next page for additional authors

Follow this and additional works at: https://digitalcommons.unl.edu/usnavyresearch

Syafruddin, Din; Asih, Puji B. S.; Casey, Gerard J.; Maguire, Jason; Baird, J. Kevin; Nagesha, Hadya S.;

Cowman, Alan F.; and Reeder, John C., "Molecular Epidemiology of Plasmodium falciparum Resistance to Antimalarial Drugs in Indonesia" (2005). U.S. Navy Research. 59.

https://digitalcommons.unl.edu/usnavyresearch/59

This Article is brought to you for free and open access by the U.S. Department of Defense at DigitalCommons@University of Nebraska - Lincoln. It has been accepted for inclusion in U.S. Navy Research by an authorized administrator of DigitalCommons@University of Nebraska - Lincoln. 


\section{Authors}

Din Syafruddin, Puji B. S. Asih, Gerard J. Casey, Jason Maguire, J. Kevin Baird, Hadya S. Nagesha, Alan F. Cowman, and John C. Reeder 


\title{
MOLECULAR EPIDEMIOLOGY OF PLASMODIUM FALCIPARUM RESISTANCE TO ANTIMALARIAL DRUGS IN INDONESIA
}

\author{
DIN SYAFRUDDIN, PUJI B. S. ASIH, GERARD J. CASEY, JASON MAGUIRE, J. KEVIN BAIRD, HADYA S. NAGESHA, \\ ALAN F. COWMAN, AND JOHN C. REEDER \\ Eijkman Institute for Molecular Biology, Jakarta, Indonesia; Department of Parasitology, Faculty of Medicine, Hasanuddin \\ University, Makassar, Indonesia; United States Naval Medical Research Unit No. 2, Jakarta, Indonesia; The Walter and Eliza Hall \\ Institute of Medical Research, Melbourne, Victoria, Australia, Papua New Guinea Institute of Medical Research, \\ Goroka, Papua New Guinea
}

\begin{abstract}
The extent of gene polymorphisms associated with resistance to chloroquine and sulfadoxinepyrimethamine was examined in field isolates of Plasmodium falciparum from Indonesia. Eight malaria-endemic areas, representing a broad region of the western and eastern Indonesian Archipelago were surveyed. Blood from 20-50 patients was collected at each site, DNA was isolated, and the sequences of four different genes (dihydrofolate reductase $[d h f r]$, dihydropteroate synthase [dhps], $P$. falciparum multidrug resistance 1 [pfmdrl], and $P$. falciparum chloroquine resistance transporter $[p f c r t]$ ) were analyzed using polymerase chain reaction and restriction fragment length polymorphisms to detect polymorphisms previously shown to be associated with resistance. This analysis identified polymorphisms in $d h f r$ at 108-Asn/Thr, 16-Val, and 59-Arg. Polymorphisms in dhps were found less frequently, either 437-Gly alone or paired with 540-Glu. The pfcrt 76-Thr polymorphism was fixed in all parasite populations and $p f m d r 186-T y r$ polymorphisms in all populations except in the most eastern regions. The pfmdr1 1042-Asp polymorphism occurred less frequently. These findings indicate that polymorphisms in genes associated with drug resistance in $P$. falciparum are found across a broad region of Indonesia.
\end{abstract}

\section{INTRODUCTION}

Since the first reports of chloroquine resistance in East Kalimantan and Indonesian Papua in $1975,{ }^{1}$ chloroquine resistance has been observed in all areas of the archipelago where in vivo/in vitro studies have been conducted. ${ }^{2-12}$ As a consequence of this widespread resistance, a combination of the antifolates sulfadoxine and pyrimethamine was introduced as a second-line treatment of simple malaria. The synergistic combination of the two, which inhibit dihydropteroate synthase and dihydrofolate reductase, respectively, in the folate biosynthetic pathway, was believed to enhance their antimalarial potency and reduce the risk of drug resistance. ${ }^{13,14}$ However, resistance to this drug combination had already been observed in Indonesia, ${ }^{1}$ and has now spread across the archipelago. , $8,10,15-17$ The resistance of malaria parasites to Fansidar ${ }^{\circledR}$ (Fansidar Hoffmann La Roche, Basel, Switzerland), the most commonly used combination of sulfadoxine-pyrimethamine, is now widespread in southeast Asia. ${ }^{18-22}$ Despite the spread of resistance to these antimalarials in Indonesia, chloroquine and sulfadoxinepyrimethamine are still used as first-line and second-line antimalarial drugs, respectively.

Molecular studies over the last few decades have identified several mutations associated with chloroquine and sulfadoxine-pyrimethamine resistance in a number of Plasmodium falciparum genes. Polymorphisms in the $P$. falciparum chloroquine resistance transporter ( $p f c r t$ ) gene, located on chromosome 7, were proposed to be important in chloroquine resistance and transfection experiments have shown that the polymorphism 76-Ser to Thr is tightly linked to the resistance phenotype. ${ }^{23,24}$ Additionally, polymorphisms in the $P$. falciparum multidrug resistance 1 ( $p f m d r 1$ ) gene have been shown by transfection to modulate higher levels of chloroquine resistance and also to affect mefloquine, halofantrine, and quinine resistance. ${ }^{25,26}$

The molecular basis of resistance to pyrimethamine and sulfadoxine has been more clearly defined. Polymorphisms in the dihydrofolate reductase $(d f h r)$ gene that alter 108 -Ser to
Asn/Thr in the enzyme have been shown to confer resistance to pyrimethamine. ${ }^{27}$ Additional polymorphisms at amino acid positions 50, 51, 59, and 164 combined with 108-Asn confer increasing levels of pyrimethamine resistance. ${ }^{28}$ The combination of 16-Ala to $16-$ Val and $108-$ Ser to 108 -Thr confers resistance to cycloguanil but retains sensitivity to pyrimethamine. ${ }^{29,30}$ Similarly, polymorphisms in the dihydropteroate synthase (dhps) gene confer resistance to sulfadoxine. ${ }^{31}$ The polymorphism 437-Gly in dhps appears to be the first to be selected by drug pressure and it encodes lower level resistance to sulfadoxine. Subsequent polymorphisms at positions $436,540,581$, and 613 confer increasing levels of resistance to this drug. ${ }^{32}$

Epidemiologic studies have been conducted in all malaria endemic areas of the world looking at polymorphisms in the aforementioned genes and their relationship with treatment failure or resistant $P$. falciparum. ${ }^{7,17,33-37}$ The aim of the present study was to complement existing knowledge of in vivo and in vitro antimalarial drug responses in Indonesia by determining the extent of associated gene polymorphisms in $P$. falciparum isolates from different malaria-endemic areas. The information obtained will contribute to the development of strategies for therapeutic intervention of malaria in Indonesia.

\section{MATERIALS AND METHODS}

Study sites. Eight malaria-endemic areas that represent the entire Indonesia archipelago (Figure 1) were selected for sample collection. Four areas (Nias, Lampung, Kokap, and Kutai) are located in western Indonesia and four (Minahasa Mamuju, Flores, and Armopa) in eastern Indonesia. In each area, 20-50 blood samples infected with $P$. falciparum isolates were collected from people either with malaria fever or apparently healthy individuals. In these selected areas, chloroquine is used as the first-line antimalarial drug and sulfadoxine-pyrimethamine is used as the second-line antimalarial drug. Primaquine, in combination with either of the above, is used for radical cure. Parenteral quinine is exclusively used as 


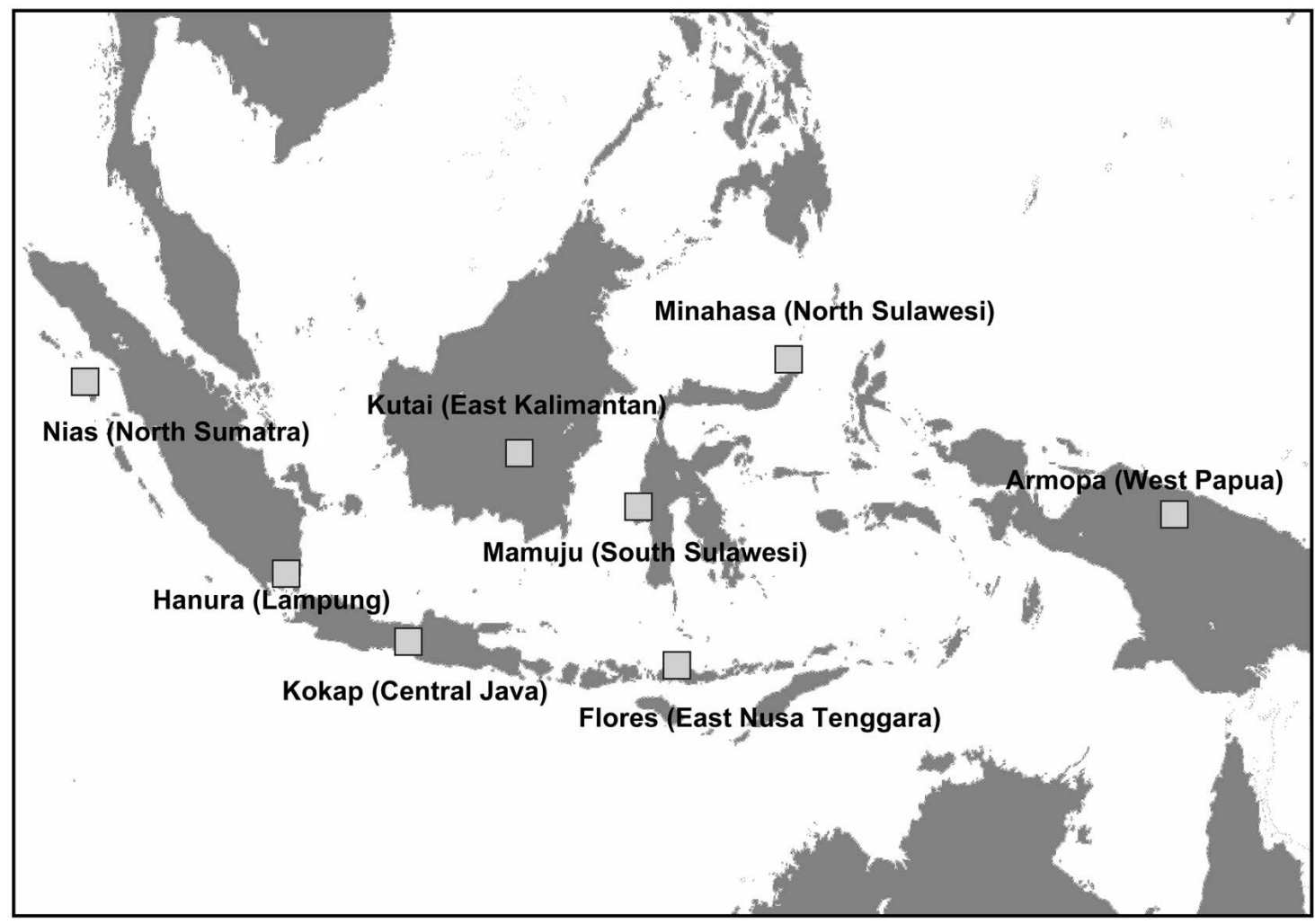

FIGURE 1. Map of the Indonesian archipelago and the sampling sites (boxes) of the isolates of Plasmodium falciparum.

a life-saving antimalarial drug in severe and complicated malaria. This study was carried out with the approval of the Ethics Committees for Protection of Human Subjects at the Eijkman Institute for Molecular Biology (Jakarta, Indonesia) and The Walter and Eliza Hall Institute (Melbourne, Victoria, Australia).

Sample collection. A malariometric survey was conducted in a selected village in each area, and blood samples were collected via one or more of the following methods: in anticoagulant tubes, smears on a glass slide, or as a blot on filter paper (3MM; Whatman, Hillsboro, OR). Plasmodium falciparum-infected samples, as revealed by microscopic examination of a slide smear, were used for DNA isolation.

Extraction of DNA. Parasite DNA was extracted from the blood samples using Chelex-100 ion exchanger (Bio-Rad Laboratories, Hercules, CA) essentially according to the procedure described previously. ${ }^{38}$ The DNA was either used immediately for a polymerase chain reaction (PCR) or stored at $-20^{\circ} \mathrm{C}$ for later analysis.

Polymerase chain reaction amplification. Nested PCRs were performed for four genes: $d h f r, d h p s, p f m d r 1$, and $p f c r t$. All reactions were carried out in $50-\mu \mathrm{L}$ reaction mixtures containing $50 \mathrm{mM} \mathrm{KCl}, 10 \mathrm{mM}$ Tris- $\mathrm{HCl}, \mathrm{pH} 8.3,1.5 \mathrm{mM}$ $\mathrm{MgCl}_{2}, 200 \mathrm{mM}$ dNTP, 1 unit of Taq polymerase (Sigma, St. Louis, MO), and a pair of primers (20 pM each). One to five microliters of DNA was used as template in the first reaction and $1-2 \mu \mathrm{L}$ of the first-round PCR product was used as template for the secondary PCR. Positive (D10 DNA) and negative (water) controls were used in all PCRs. The PCR primers and conditions were as previously described for $d h f r, d h p s$, and $p f m d r 1,{ }^{39,40}$ and $p f c r t .^{23,24}$ Secondary PCR products were resolved by electrophoresis on 1-2\% agarose gels and visualized by staining with ethidium bromide.

Restriction fragment length polymorphism (RFLP). Restriction enzyme digestion of either the native or introduced sites of the PCR products was performed to determine the presence of polymorphisms in $d h f r$, dhps, pfmdr $1,{ }^{39-42}$ and pfcrt. ${ }^{23-25}$ A number of restriction enzymes were used for RFLP of PCR products. For $d h f r$, the PCR products were digested with Nla III, Tai I, Tsp 509I, Xmn I, and Dra I to determine the polymorphisms at codons $16,50,51,59$, and 164, respectively. Three enzymes, Alu I, Bsr I, and Scr FI, were used to identify codon 108 . For $d h p s$, the PCR products were digested with Msp A1I, Ava II, and Fok I to determine the polymorphisms at codon 436,437 , and 540 , respectively. In addition, restriction enzymes $B s t$ UI and $B s l$ I were used to identify polymorphisms at codon 581, while Mwo I, Bsa WI, and Age I were used for codon 613. Similarly, pfmdr1 PCR products were digested with $A f l \mathrm{III}, D d e$ I, Ase I, and Eco RV to determine the presence of polymorphisms at codons 86 , 1034, 1042, and 1246, respectively. For $p f c r t$ PCR products, the polymorphism at codon 76 was examined by digestion with Apo I. To determine complete digestion, introduced or natural restriction sites in the DNA fragment served as controls. If no such restriction sites were present, a known PCR product carrying the required restriction sites was used. As an additional control in every RFLP experiment, $0.5 \mu \mathrm{g}$ of purified lambda DNA (Promega, Madison, WI) was digested to monitor the enzyme activity. Digested products were subjected to electrophoresis on 1.5-3\% agarose gels (Progen, Australia) or $1.5-3 \%$ ultrapure agarose gels (Progen, Australia) when a higher resolution was required. Loss of restriction 
sites in the DNA fragment for the enzymes Nla III, Tsp 509I, Dde I, and Ase I or a gain in the restriction sites for Tai I, $X m n$ I, Bsr I, Scr FI, Dra I, Msp A1I, Ava II, Fok I, Bsl I, Bsa WI, Age I, Afl III, and Eco RV indicated that the DNA fragments carried polymorphisms. If there was an indication of incomplete digestion in a sample, the digestion were repeated with overnight incubation to confidently accept the presence of a mixed allelic population. These cases were considered to be mixed infections.

\section{RESULTS}

A total of 213 P. falciparum isolates from eight malariaendemic areas in Indonesia were analyzed. Some samples, particularly those collected on filter paper, failed to amplify using certain primers; thus, the gene polymorphism information obtained was incomplete. Based on gene analysis, there were 83 samples $(38.9 \%)$ that gave PCR results indicating mixed isolates, either as polymorphic and wild-type or two polymorphic types.

Genotypic profiles of $\boldsymbol{P}$. falciparum isolates from western Indonesia. Polymorphisms in the pfmdr1 and pfcrt genes. Analysis of 117 isolates from Nias Island, Hanura, Kokap, and Kutai showed that polymorphisms in the $p f m d r 1$ and pfcrt genes have spread to all sample collection sites (Table 1). Except in one sample from Nias Island that failed to be amplified, all (116 of 116) isolates carried the $86 Y$ polymorphism in pfmdr1. No polymorphisms at codons 1034, 1042 , and 1246 of the $p f m d r 1$ gene were observed in any of the isolates examined. Likewise, for $p f c r t, 100 \%$ ( 85 of 85 ) of the amplified samples carried the $76 T$ polymorphism.

Polymorphisms in the dhfr and dhps genes. Amplification of $d h f r 108$ was successful with 92 isolates (Table 1). Of these, $54 \%$ (50 of 92) had either a single polymorphism, $108 \mathrm{~N}$, or the mixed S108N and 19\% (17 of 92) had either $108 \mathrm{~T}$ or $108 \mathrm{~T}$ mixed with wild-type. An additional 13\% (12 of 92) carried the mixture 108N/T. Except for one isolate from Kutai, the threonine polymorphism was found only at Hanura and Kokap. Only $14 \%$ (13 of 92) of the successfully amplified isolates for $d h f r 108$ carried a single wild-type haplotype. Analysis of codon 59 was successful in 90 of the 92 amplified isolates. This identified 54\% (49 of 90) as being wild-type C59 while the other $46 \%$ (41 of 90) were either 59R or 59R mixed with wild-type. The C59R polymorphism was in all cases associated with one or other of the 108 resistance polymorphisms. At Kutai, the 59R polymorphism was not observed. At $d h f r$ codon 16, the wild-type A16 was observed in 62\% (16 of 26) of isolates from Kokap and the single polymorphism $16 \mathrm{~V}$ or the mixed A16V was observed in $38 \%$ (10 of 26 ) of the isolates. This polymorphism was only observed in association with $108 \mathrm{~T}$ as either a single or mixed population. Codon 16 was only observed as wild-type at all other locations in the western part of Indonesia. No polymorphisms were observed at codons 50, 51, or 164 in any of the samples examined.

Polymorphisms in the dhps gene were the least common among the four genes tested in this study (Table 1). The most common polymorphism was $437 \mathrm{G}$, which was observed in Hanura, Kokap, and Kutai; however, only in 14\% (16 of 117) of all isolates. The double polymorphism of codons $437 \mathrm{G}$ and $540 \mathrm{E}$ was observed in one sample from Kokap, in association with $d h f r$ 59R and 108N/T. No polymorphisms were observed at codons 436, 581, or 613 in any of the samples examined in this study.

Genotypic profiles of $\boldsymbol{P}$. falciparum isolates from eastern Indonesia. Analysis of pfmdr1 and pfcrt genes. Analysis of 71 isolates that successfully amplified pfcrt codon 76 identified $91 \%$ (64 of 70) with the polymorphism 76T and one isolate from each of Flores and Armopa were mixed K76T (Table 2). For $p f m d r 1$, the results were more complex, with the $86 \mathrm{Y}$ polymorphism ranging from $95 \%$ (19 of 20) at Minahasa to $16 \%$ (3 of 19) at Flores. However, the $84 \%$ (16 of 19) of isolates from Flores that carried the wild-type N86 were observed to have the polymorphism 1042D. At Armopa, 27\% (4 of 15 ) of the isolates carried the $86 \mathrm{Y}$ polymorphism and all isolates were wild-type at codon 1042 . The $1042 \mathrm{D}$ polymorphism was also observed in 15\% (3 of 20) of isolates from Minahasa.

Point mutations in the dhfr and dhps genes. Eighty-three samples were successfully amplified at $d h f r 108$, of which only $10 \%$ (8 of 83 ) were wild-type S108. The rest were either $108 \mathrm{~T}$ (34\%, 28 of 83), S108T (20\%, 17 of 83), 108N (19\%, 16 of 83), S108N (12\%, 10 of 83) or 108N/T (5\%, 4 of 83). Eighty isolates were successfully analyzed for polymorphisms at codon 59. A total of $68 \%$ (54 of 80) were wild-type C59 and 32\% (26 of 80 ) were either $59 \mathrm{R}$ or the mixed $59 \mathrm{R}$ with wild-type (Table 2). At codon 16, the polymorphism $16 \mathrm{~V}$ was observed twice at Mamuju, once in association with $108 \mathrm{~T}$ and once with 59R and 108T. In Flores, 16V was observed once in association with $108 \mathrm{~T}$ and once as the mixed A16V in association with S108T. No polymorphisms were observed at codons 50, 51, and 164 in any of the samples examined.

Analysis of dhps showed that 13\% (13 of 96) of the isolates were either 437G or A437G. One isolate from Minahasa carried the $540 \mathrm{E}$ polymorphism associated with $437 \mathrm{G}$ and $d h f r$ S108T. A single isolate from Armopa carried dhps K540G in association with A437G and $d h f r$ C59 and S108. No mutation was observed at codons 436, 581, and 613 in any of the samples examined in this study.

\section{DISCUSSION}

As might have been expected from the previous extensive studies on in vivo and in vitro antimalarial drug responses in Indonesia, the isolates of $P$. falciparum examined in this study were found to carry multiple genetic polymorphisms associated with resistance to chloroquine and sulfadoxinepyrimethamine.

Although the molecular basis for the $P$. falciparum resistance to quinoline antimalarials is still being investigated, evidence indicates that resistance is multigenic. Initially, the role of mutations in pfmdr1 in the modulation of chloroquine resistance was shown. ${ }^{27}$ Allelic exchange experiments have indicated that the 1246 Tyr polymorphism in $p f m d r 1$ increases the chloroquine $50 \%$ inhibitory concentration $\left(\mathrm{IC}_{50}\right)$ and, both alone and in combination with the polymorphisms 1034 Cys and 1042 Asp, also modulates sensitivity to other antimalarials drugs. ${ }^{28}$ However, a detailed linkage analysis and chromosomal mapping of progeny identified a second gene involved in chloroquine resistance, $p f c r t$, in which mutation at codon $76, \mathrm{~K} 76 \mathrm{~T}$ is strongly associated with the chloroquine resistance phenotypes in field and clinical studies. ${ }^{23-25}$ Furthermore, conclusive evidence that various mutant $p f c r t$ hap- 
EPIDEMIOLOGY OF P. FALCIPARUM DRUG RESISTANCE IN INDONESIA

TABLE 1

Genotypic pattern of Plasmodium falciparum isolates from western Indonesia*

\begin{tabular}{|c|c|c|c|c|c|c|c|c|c|c|}
\hline \multirow[b]{2}{*}{ Locations } & \multirow{2}{*}{$\begin{array}{l}\text { No. of } \\
\text { genotypes }\end{array}$} & \multicolumn{3}{|c|}{ DHFR } & \multicolumn{2}{|c|}{ DHPS } & \multicolumn{2}{|c|}{$P f m d r 1$} & \multirow{2}{*}{$\begin{array}{l}P f C R T \\
\quad 76 T\end{array}$} & \multirow{2}{*}{$\begin{array}{l}\text { No. of } \\
\text { isolates }\end{array}$} \\
\hline & & $16 \mathrm{~V}$ & $59 R$ & $108 N / T$ & $437 G$ & $540 E$ & $86 Y$ & $1042 \mathrm{D}$ & & \\
\hline \multirow{8}{*}{ Nias, North Sumatra } & 1. & A & $\mathrm{C}$ & $\mathrm{S}$ & A & $\mathrm{K}$ & $\mathrm{Y}$ & $\mathrm{N}$ & $\mathrm{T}$ & 1 \\
\hline & 2. & A & $\mathrm{C}$ & $\mathrm{N}$ & A & $\mathrm{K}$ & $\mathrm{Y}$ & $\mathrm{N}$ & $\mathrm{T}$ & 2 \\
\hline & 3. & A & $\mathrm{R}$ & $\mathrm{N}$ & A & $\mathrm{K}$ & $\mathrm{Y}$ & $\mathrm{N}$ & $\mathrm{T}$ & 9 \\
\hline & 4. & A & $\mathrm{C} / \mathrm{R}$ & $\mathrm{S} / \mathrm{N}$ & A & $\mathrm{K}$ & $\mathrm{Y}$ & $\mathrm{N}$ & $\mathrm{T}$ & 3 \\
\hline & 5. & A & - & $\mathrm{N}$ & A & $\mathrm{K}$ & $\mathrm{Y}$ & $\mathrm{N}$ & $\mathrm{T}$ & 1 \\
\hline & 6. & A & - & $\mathrm{S} / \mathrm{N}$ & A & $\mathrm{K}$ & $\mathrm{Y}$ & $\mathrm{N}$ & $\mathrm{T}$ & 1 \\
\hline & 7. & - & - & - & A & $\mathrm{K}$ & $\mathrm{Y}$ & $\mathrm{N}$ & $\mathrm{T}$ & 2 \\
\hline & 8. & - & - & - & A & $\mathrm{K}$ & - & - & $\mathrm{T}$ & 1 \\
\hline \multirow[t]{16}{*}{ Hanura, Lampung } & 1. & A & $\mathrm{R}$ & $\mathrm{N}$ & A & $\mathrm{K}$ & $\mathrm{Y}$ & $\mathrm{N}$ & $\mathrm{T}$ & 3 \\
\hline & 2. & A & $\mathrm{R}$ & $\mathrm{S} / \mathrm{N}$ & A & $\mathrm{K}$ & $\mathrm{Y}$ & $\mathrm{N}$ & $\mathrm{T}$ & 2 \\
\hline & 3. & A & $\mathrm{R}$ & $\mathrm{N}$ & $\mathrm{A} / \mathrm{G}$ & $\mathrm{K}$ & $\mathrm{Y}$ & $\mathrm{N}$ & $\mathrm{T}$ & 2 \\
\hline & 4. & A & $\mathrm{C}$ & $\mathrm{S} / \mathrm{N}$ & A & $\mathrm{K}$ & $\mathrm{Y}$ & $\mathrm{N}$ & $\mathrm{T}$ & 4 \\
\hline & 5. & A & $\mathrm{R}$ & $\mathrm{N}$ & $\mathrm{A} / \mathrm{G}$ & $\mathrm{K}$ & $\mathrm{Y}$ & $\mathrm{N}$ & $\mathrm{T}$ & 3 \\
\hline & 6. & A & $\mathrm{C}$ & $\mathrm{S} / \mathrm{N}$ & $\mathrm{A} / \mathrm{G}$ & $\mathrm{K}$ & $\mathrm{Y}$ & $\mathrm{N}$ & $\mathrm{T}$ & 1 \\
\hline & 7. & A & $\mathrm{R}$ & $\mathrm{N} / \mathrm{T}$ & A & $\mathrm{K}$ & $\mathrm{Y}$ & $\mathrm{N}$ & $\mathrm{T}$ & 1 \\
\hline & 8. & A & $\mathrm{C} / \mathrm{R}$ & $\mathrm{N} / \mathrm{T}$ & A & $\mathrm{K}$ & $\mathrm{Y}$ & $\mathrm{N}$ & $\mathrm{T}$ & 2 \\
\hline & 9. & A & $\mathrm{C} / \mathrm{R}$ & N/T & $\mathrm{G}$ & $\mathrm{K}$ & $\mathrm{Y}$ & $\mathrm{N}$ & $\mathrm{T}$ & 2 \\
\hline & 10. & A & $\mathrm{C} / \mathrm{R}$ & $\mathrm{N} / \mathrm{T}$ & $\mathrm{A} / \mathrm{G}$ & $\mathrm{K}$ & $\mathrm{Y}$ & $\mathrm{N}$ & $\mathrm{T}$ & 3 \\
\hline & 11. & A & $\mathrm{C} / \mathrm{R}$ & $\mathrm{S} / \mathrm{N}$ & $\mathrm{A} / \mathrm{G}$ & $\mathrm{K}$ & $\mathrm{Y}$ & $\mathrm{N}$ & $\mathrm{T}$ & 1 \\
\hline & 12. & A & $\mathrm{C}$ & $\mathrm{S}$ & A & $\mathrm{K}$ & $\mathrm{Y}$ & $\mathrm{N}$ & - & 3 \\
\hline & 13. & A & $\mathrm{C}$ & $\mathrm{T}$ & A & $\mathrm{K}$ & $\mathrm{Y}$ & $\mathrm{N}$ & - & 2 \\
\hline & 14. & A & $\mathrm{R}$ & $\mathrm{N}$ & A & $\mathrm{K}$ & $\mathrm{Y}$ & $\mathrm{N}$ & - & 1 \\
\hline & 15. & - & - & - & A & $\mathrm{K}$ & $\mathrm{Y}$ & $\mathrm{N}$ & $\mathrm{T}$ & 1 \\
\hline & 16. & - & - & - & A & $\mathrm{K}$ & $\mathrm{Y}$ & $\mathrm{N}$ & - & 10 \\
\hline \multirow[t]{20}{*}{ Kokap, Central Java } & 1. & A & $\mathrm{C}$ & $\mathrm{S}$ & A & $\mathrm{K}$ & $\mathrm{Y}$ & $\mathrm{N}$ & $\mathrm{T}$ & 2 \\
\hline & 2. & A & $\mathrm{R}$ & $\mathrm{N}$ & A & $\mathrm{K}$ & $\mathrm{Y}$ & $\mathrm{N}$ & $\mathrm{T}$ & 3 \\
\hline & 3. & A & $\mathrm{C}$ & $\mathrm{T}$ & A & $\mathrm{K}$ & $\mathrm{Y}$ & $\mathrm{N}$ & $\mathrm{T}$ & 2 \\
\hline & 4. & V & $\mathrm{C}$ & $\mathrm{T}$ & A & $\mathrm{K}$ & $\mathrm{Y}$ & $\mathrm{N}$ & $\mathrm{T}$ & 2 \\
\hline & 5. & A & $\mathrm{C} / \mathrm{R}$ & $\mathrm{N}$ & A & $\mathrm{K}$ & $\mathrm{Y}$ & $\mathrm{N}$ & $\mathrm{T}$ & 1 \\
\hline & 6. & V & $\mathrm{C}$ & $\mathrm{N} / \mathrm{T}$ & A & $\mathrm{K}$ & $\mathrm{Y}$ & $\mathrm{N}$ & $\mathrm{T}$ & 1 \\
\hline & 7. & A & $\mathrm{R}$ & $\mathrm{N} / \mathrm{T}$ & A & $\mathrm{K}$ & $\mathrm{Y}$ & $\mathrm{N}$ & $\mathrm{T}$ & 1 \\
\hline & 8. & A & $\mathrm{R}$ & $\mathrm{N} / \mathrm{T}$ & $\mathrm{G}$ & $\mathrm{E}$ & $\mathrm{Y}$ & $\mathrm{N}$ & $\mathrm{T}$ & 1 \\
\hline & 9. & A & $\mathrm{C} / \mathrm{R}$ & $\mathrm{S} / \mathrm{N}$ & A & $\mathrm{K}$ & $\mathrm{Y}$ & $\mathrm{N}$ & $\mathrm{T}$ & 1 \\
\hline & 10. & $\mathrm{~A} / \mathrm{V}$ & $\mathrm{C}$ & $\mathrm{S} / \mathrm{T}$ & A & $\mathrm{K}$ & $\mathrm{Y}$ & $\mathrm{N}$ & $\mathrm{T}$ & 1 \\
\hline & 11. & $\mathrm{~A} / \mathrm{V}$ & $\mathrm{C} / \mathrm{R}$ & $\mathrm{N} / \mathrm{T}$ & A & $\mathrm{K}$ & $\mathrm{Y}$ & $\mathrm{N}$ & $\mathrm{T}$ & 1 \\
\hline & 12. & $\mathrm{~V}$ & $\mathrm{C}$ & $\mathrm{T}$ & A & $\mathrm{K}$ & $\mathrm{Y}$ & $\mathrm{N}$ & $\mathrm{T}$ & 3 \\
\hline & 13. & A & $\mathrm{R}$ & $\mathrm{N}$ & A & $\mathrm{K}$ & $\mathrm{Y}$ & $\mathrm{N}$ & $\mathrm{T}$ & 1 \\
\hline & 14. & A & $\mathrm{C}$ & $\mathrm{S} / \mathrm{T}$ & A & $\mathrm{K}$ & $\mathrm{Y}$ & $\mathrm{N}$ & - & 1 \\
\hline & 15. & $\mathrm{~A} / \mathrm{V}$ & $\mathrm{C}$ & $\mathrm{S} / \mathrm{T}$ & A & $\mathrm{K}$ & $\mathrm{Y}$ & $\mathrm{N}$ & - & 1 \\
\hline & 16. & A & $\mathrm{C}$ & $\mathrm{N}$ & A & $\mathrm{K}$ & $\mathrm{Y}$ & $\mathrm{N}$ & - & 1 \\
\hline & 17. & A & $\mathrm{C}$ & $\mathrm{T}$ & A & $\mathrm{K}$ & $\mathrm{Y}$ & $\mathrm{N}$ & - & 2 \\
\hline & 18. & $\mathrm{~A} / \mathrm{V}$ & $\mathrm{C}$ & $\mathrm{T}$ & A & $\mathrm{K}$ & $\mathrm{Y}$ & $\mathrm{N}$ & - & 1 \\
\hline & 19. & - & $\mathrm{C}$ & $\mathrm{T}$ & A & $\mathrm{K}$ & $\mathrm{Y}$ & $\mathrm{N}$ & - & 1 \\
\hline & 20. & - & - & - & A & $\mathrm{K}$ & $\mathrm{Y}$ & $\mathrm{N}$ & $\mathrm{T}$ & 1 \\
\hline \multirow[t]{9}{*}{ Kutai, East Kalimantan } & 1. & A & $\mathrm{C}$ & $\mathrm{S}$ & A & $\mathrm{K}$ & $\mathrm{Y}$ & $\mathrm{N}$ & $\mathrm{T}$ & 5 \\
\hline & 2. & A & $\mathrm{C}$ & $\mathrm{N}$ & A & $\mathrm{K}$ & $\mathrm{Y}$ & $\mathrm{N}$ & $\mathrm{T}$ & 5 \\
\hline & 3. & A & $\mathrm{C}$ & $\mathrm{S} / \mathrm{T}$ & A & $\mathrm{K}$ & $\mathrm{Y}$ & $\mathrm{N}$ & $\mathrm{T}$ & 1 \\
\hline & 4. & A & $\mathrm{C}$ & $\mathrm{S} / \mathrm{N}$ & A & $\mathrm{K}$ & $\mathrm{Y}$ & $\mathrm{N}$ & $\mathrm{T}$ & 3 \\
\hline & 5. & A & $\mathrm{C}$ & $\mathrm{S}$ & $\mathrm{A} / \mathrm{G}$ & $\mathrm{K}$ & $\mathrm{Y}$ & $\mathrm{N}$ & $\mathrm{T}$ & 1 \\
\hline & 6. & A & $\mathrm{C}$ & $\mathrm{N}$ & $\mathrm{A} / \mathrm{G}$ & $\mathrm{K}$ & $\mathrm{Y}$ & $\mathrm{N}$ & $\mathrm{T}$ & 2 \\
\hline & 7. & A & $\mathrm{C}$ & $\mathrm{S}$ & A & $\mathrm{K}$ & $\mathrm{Y}$ & $\mathrm{N}$ & - & 1 \\
\hline & 8. & - & - & - & A & $\mathrm{K}$ & $\mathrm{Y}$ & $\mathrm{N}$ & $\mathrm{T}$ & 2 \\
\hline & 9. & - & - & - & A & $\mathrm{K}$ & $\mathrm{Y}$ & $\mathrm{N}$ & - & 8 \\
\hline
\end{tabular}

$* D H F R=$ dihydrofolate reductase; $D H P S=$ dihydropteroate synthase; $P f m d r 1=P$. falciparum multidrug resistance $1 ;$ PfCRT $=P$. falciparum chloroquine resistance transporter.

lotypes confer chloroquine resistance with characteristic verapamil reversibility and reduced chloroquine accumulation was demonstrated. ${ }^{26}$ Nevertheless, the role of other modifying factor such as pfmdrl in this regard could not be excluded.

Previous field-based studies in Indonesia have associated the $86 \mathrm{Y}$ allele of the $p f m d r 1$ gene to chloroquine resistance, both in vivo and in vitro. ${ }^{8,41}$ Other studies in Indonesia have reported that the 76T polymorphism of $p f c r t$ is also associated with chloroquine resistance in vivo and in vitro, and the allele has the potential to be used as a predictor for chloroquine therapeutic treatment failure. ${ }^{7,42}$ Our present results show that the isolates of $P$. falciparum from Indonesia carry polymorphisms in both the pfcrt and pfmdr1 genes. In western Indonesia, all asymptomatic and mildly symptomatic malaria patients were carrying parasites with both pfcrt 76T and pfmdr1 86Y polymorphisms. The situation in eastern Indonesia was more complex (Figure 2). Northern Sulawesi had a resistant profile at these two codons, whereas southern Sulawesi had a lower frequency of $p f m d r 186 \mathrm{Y}$ polymorphism than may have been expected, but $p f c r t$ was fixed in the parasite population. In Flores, all isolates carried 76T and all isolates carried a polymorphism in $p f m d r 1$. However, most of 
TABLE 2

Genotypic pattern of Plasmodium falciparum isolates from eastern Indonesia*

\begin{tabular}{|c|c|c|c|c|c|c|c|c|c|c|}
\hline \multirow[b]{2}{*}{ Locations } & \multirow{2}{*}{$\begin{array}{c}\text { No. of } \\
\text { genotypes }\end{array}$} & \multicolumn{3}{|c|}{ DHFR } & \multicolumn{2}{|c|}{ DHPS } & \multicolumn{2}{|c|}{ Pfmdr1 } & \multirow{2}{*}{$\begin{array}{l}P f C R T \\
\quad 76 T\end{array}$} & \multirow{2}{*}{$\begin{array}{l}\text { No. of } \\
\text { isolates }\end{array}$} \\
\hline & & $\overline{16 \mathrm{~V}}$ & $59 R$ & $\overline{108 N / T}$ & $\overline{437 G}$ & $\overline{540 E}$ & $86 Y$ & $\overline{1042 D}$ & & \\
\hline \multirow{25}{*}{ Minahasa, North Sulawesi } & 1. & A & $\mathrm{C}$ & $\mathrm{T}$ & A & $\mathrm{K}$ & $\mathrm{N} / \mathrm{Y}$ & $\mathrm{N}$ & $\mathrm{K}$ & 1 \\
\hline & 2. & A & $\mathrm{C}$ & $\mathrm{N}$ & $\mathrm{A} / \mathrm{G}$ & $\mathrm{K}$ & $\mathrm{Y}$ & $\mathrm{N}$ & $\mathrm{T}$ & 1 \\
\hline & 3. & A & $\mathrm{C}$ & $\mathrm{S} / \mathrm{N}$ & A & $\mathrm{K}$ & $\mathrm{Y}$ & $\mathrm{N}$ & $\mathrm{T}$ & 2 \\
\hline & 4. & A & $\mathrm{C}$ & $\mathrm{S} / \mathrm{N}$ & $\mathrm{G}$ & $\mathrm{K}$ & $\mathrm{Y}$ & $\mathrm{N}$ & $\mathrm{T}$ & 1 \\
\hline & 5. & A & $\mathrm{C} / \mathrm{R}$ & $\mathrm{N} / \mathrm{T}$ & A & $\mathrm{K}$ & $\mathrm{Y}$ & $\mathrm{N}$ & $\mathrm{T}$ & 1 \\
\hline & 6. & A & $\mathrm{C}$ & $\mathrm{S} / \mathrm{N}$ & A & $\mathrm{K}$ & $\mathrm{N} / \mathrm{Y}$ & $\mathrm{N}$ & - & 1 \\
\hline & 7. & A & $\mathrm{C}$ & $\mathrm{S} / \mathrm{N}$ & $\mathrm{G}$ & $\mathrm{K}$ & $\mathrm{Y}$ & $\mathrm{N}$ & - & 1 \\
\hline & 8. & A & $\mathrm{C}$ & $\mathrm{S} / \mathrm{T}$ & A & $\mathrm{K}$ & $\mathrm{Y}$ & $\mathrm{D}$ & - & 1 \\
\hline & 9. & A & $\mathrm{C}$ & $\mathrm{S} / \mathrm{T}$ & $\mathrm{G}$ & $\mathrm{K}$ & $\mathrm{Y}$ & $\mathrm{D}$ & - & 1 \\
\hline & 10. & A & $\mathrm{C}$ & $\mathrm{S} / \mathrm{T}$ & $\mathrm{G}$ & $\mathrm{E}$ & $\mathrm{N}$ & $\mathrm{D}$ & - & 1 \\
\hline & 11. & A & $\mathrm{C}$ & $\mathrm{N} / \mathrm{T}$ & A & $\mathrm{K}$ & $\mathrm{Y}$ & - & $\mathrm{T}$ & 1 \\
\hline & 12. & A & $\mathrm{C}$ & $\mathrm{N}$ & A & K & - & $\mathrm{N}$ & $\mathrm{T}$ & 1 \\
\hline & 13. & A & $\mathrm{C}$ & $\mathrm{S} / \mathrm{T}$ & A & $\mathrm{K}$ & - & - & $\mathrm{T}$ & 2 \\
\hline & 14. & A & $\mathrm{C}$ & $\mathrm{N} / \mathrm{T}$ & A & $\mathrm{K}$ & - & - & $\mathrm{T}$ & 1 \\
\hline & 15. & A & $\mathrm{C} / \mathrm{R}$ & $\mathrm{N} / \mathrm{T}$ & A & $\mathrm{K}$ & - & - & $\mathrm{T}$ & 1 \\
\hline & 16. & A & $\mathrm{C}$ & $\mathrm{S} / \mathrm{T}$ & A & $\mathrm{K}$ & - & $\mathrm{N}$ & - & 2 \\
\hline & 17. & A & $\mathrm{C}$ & $\mathrm{S} / \mathrm{T}$ & A & $\mathrm{K}$ & - & - & - & 3 \\
\hline & 18. & - & - & - & A & $\mathrm{K}$ & $\mathrm{Y}$ & $\mathrm{N}$ & $\mathrm{T}$ & 3 \\
\hline & 19. & - & - & - & A & $\mathrm{K}$ & $\mathrm{Y}$ & $\mathrm{N}$ & - & 1 \\
\hline & 20. & - & - & - & $\mathrm{A} / \mathrm{G}$ & $\mathrm{K}$ & $\mathrm{Y}$ & $\mathrm{N}$ & - & 1 \\
\hline & 21. & - & - & - & A & K & - & $\mathrm{N}$ & $\mathrm{T}$ & 1 \\
\hline & 22. & - & - & - & A & $\mathrm{K}$ & $\mathrm{Y}$ & - & $\mathrm{T}$ & 2 \\
\hline & 23. & - & - & - & $\mathrm{A} / \mathrm{G}$ & K & - & $\mathrm{N}$ & - & 1 \\
\hline & 24 & - & - & _ & A & $\mathrm{K}$ & - & $\mathrm{N}$ & - & 2 \\
\hline & 25. & - & - & - & $\mathrm{A} / \mathrm{G}$ & $\mathrm{K}$ & $\mathrm{Y}$ & - & - & 1 \\
\hline \multirow[t]{13}{*}{ Mamuju, South Sulawesi } & 1. & A & $\mathrm{C}$ & $\mathrm{T}$ & A & $\mathrm{K}$ & $\mathrm{Y}$ & $\mathrm{N}$ & $\mathrm{T}$ & 2 \\
\hline & 2. & $\mathrm{~V}$ & $\mathrm{R}$ & $\mathrm{T}$ & A & $\mathrm{K}$ & $\mathrm{Y}$ & $\mathrm{N}$ & $\mathrm{T}$ & 1 \\
\hline & 3. & A & $\mathrm{C}$ & $\mathrm{N}$ & A & $\mathrm{K}$ & $\mathrm{N}$ & $\mathrm{N}$ & $\mathrm{T}$ & 4 \\
\hline & 4. & A & $\mathrm{C}$ & $\mathrm{N}$ & A & $\mathrm{K}$ & $\mathrm{Y}$ & $\mathrm{N}$ & $\mathrm{T}$ & 1 \\
\hline & 5. & A & $\mathrm{C}$ & $\mathrm{S} / \mathrm{N}$ & $\mathrm{G}$ & $\mathrm{K}$ & $\mathrm{N}$ & $\mathrm{N}$ & $\mathrm{T}$ & 1 \\
\hline & 6. & A & $\mathrm{C}$ & $\mathrm{T}$ & A & $\mathrm{K}$ & $\mathrm{Y}$ & $\mathrm{N}$ & - & 5 \\
\hline & 7. & A & $\mathrm{C} / \mathrm{R}$ & $\mathrm{T}$ & A & $\mathrm{K}$ & $\mathrm{Y}$ & $\mathrm{N}$ & - & 1 \\
\hline & 8. & A & $\mathrm{C}$ & $\mathrm{T}$ & A & $\mathrm{K}$ & - & - & $\mathrm{T}$ & 2 \\
\hline & 9. & A & $\mathrm{C} / \mathrm{R}$ & $\mathrm{T}$ & A & K & - & - & $\mathrm{T}$ & 1 \\
\hline & 10. & V & $\mathrm{C}$ & $\mathrm{T}$ & A & K & - & - & $\mathrm{T}$ & 1 \\
\hline & 11. & A & $\mathrm{R}$ & $\mathrm{T}$ & A & $\mathrm{K}$ & - & - & $\mathrm{T}$ & 2 \\
\hline & 12. & A & $\mathrm{C}$ & $\mathrm{T}$ & A & K & - & - & - & 1 \\
\hline & 13. & - & - & - & A & $\mathrm{K}$ & $\mathrm{N}$ & $\mathrm{N}$ & $\mathrm{T}$ & 1 \\
\hline \multirow[t]{13}{*}{ Flores, East Nusa Tenggara } & 1. & A & $\mathrm{R}$ & $\mathrm{T}$ & A & $\mathrm{K}$ & $\mathrm{N}$ & $\mathrm{D}$ & $\mathrm{T}$ & 2 \\
\hline & 2. & A & $\mathrm{C}$ & S & A & $\mathrm{K}$ & $\mathrm{Y}$ & $\mathrm{N}$ & $\mathrm{T}$ & 1 \\
\hline & 3. & $\mathrm{~V}$ & $\mathrm{C}$ & $\mathrm{T}$ & A & $\mathrm{K}$ & $\mathrm{N}$ & $\mathrm{D}$ & $\mathrm{T}$ & 1 \\
\hline & 4. & A & $\mathrm{C}$ & $\mathrm{T}$ & A & $\mathrm{K}$ & $\mathrm{N}$ & $\mathrm{D}$ & $\mathrm{T}$ & 5 \\
\hline & 5. & A & $\mathrm{C}$ & $\mathrm{T}$ & A & $\mathrm{K}$ & $\mathrm{Y}$ & $\mathrm{N}$ & $\mathrm{T}$ & 1 \\
\hline & 6. & A & C & $S$ & A & $\mathrm{K}$ & $\mathrm{N}$ & $\mathrm{D}$ & $\mathrm{T}$ & 1 \\
\hline & 7. & A & $\mathrm{C}$ & $\mathrm{S} / \mathrm{T}$ & A & $\mathrm{K}$ & $\mathrm{N}$ & $\mathrm{D}$ & $\mathrm{T}$ & 1 \\
\hline & 8. & A & $\mathrm{R}$ & $\mathrm{S} / \mathrm{T}$ & A & $\mathrm{K}$ & $\mathrm{N}$ & $\mathrm{D}$ & $\mathrm{T}$ & 3 \\
\hline & 9. & A & $\mathrm{C}$ & $\mathrm{T}$ & A & $\mathrm{K}$ & $\mathrm{N}$ & $\mathrm{D}$ & $\mathrm{K} / \mathrm{T}$ & 1 \\
\hline & 10. & A & $\mathrm{C} / \mathrm{R}$ & $\mathrm{S} / \mathrm{T}$ & A & $\mathrm{K}$ & $\mathrm{N} / \mathrm{Y}$ & $\mathrm{N} / \mathrm{D}$ & $\mathrm{T}$ & 1 \\
\hline & 11. & A & - & $\mathrm{S} / \mathrm{T}$ & A & $\mathrm{K}$ & $\mathrm{N}$ & $\mathrm{D}$ & $\mathrm{T}$ & 1 \\
\hline & 12. & $\mathrm{~A} / \mathrm{V}$ & - & $\mathrm{S} / \mathrm{T}$ & A & $\mathrm{K}$ & $\mathrm{N}$ & $\mathrm{D}$ & $\mathrm{T}$ & 1 \\
\hline & 13. & A & - & $\mathrm{T}$ & A & $\mathrm{K}$ & - & - & $\mathrm{T}$ & 1 \\
\hline \multirow[t]{13}{*}{ Armopa, West Papua } & 1. & A & $\mathrm{C}$ & $S$ & $\mathrm{~A} / \mathrm{G}$ & $\mathrm{K} / \mathrm{E}$ & $\mathrm{N}$ & $\mathrm{N}$ & $\mathrm{T}$ & 1 \\
\hline & 2. & A & $\mathrm{C} / \mathrm{R}$ & $\mathrm{S} / \mathrm{N}$ & A & K & $\mathrm{N}$ & $\mathrm{N}$ & $\mathrm{T}$ & 3 \\
\hline & 3. & A & $\mathrm{R}$ & $\mathrm{N}$ & A & $\mathrm{K}$ & $\mathrm{N}$ & $\mathrm{N}$ & $\mathrm{T}$ & 2 \\
\hline & 4. & A & $\mathrm{R}$ & $\mathrm{N}$ & $\mathrm{G}$ & K & $\mathrm{N}$ & $\mathrm{N}$ & $\mathrm{T}$ & 1 \\
\hline & 5. & A & $\mathrm{C}$ & $\mathrm{S}$ & A & $\mathrm{K}$ & $\mathrm{N}$ & $\mathrm{N}$ & $\mathrm{T}$ & 2 \\
\hline & 6. & A & $\mathrm{R}$ & $\mathrm{N}$ & A & $\mathrm{K}$ & $\mathrm{N} / \mathrm{Y}$ & $\mathrm{N}$ & $\mathrm{K} / \mathrm{T}$ & 1 \\
\hline & 7. & A & $\mathrm{C}$ & $\mathrm{S}$ & A & $\mathrm{K}$ & $\mathrm{N}$ & $\mathrm{N}$ & $\mathrm{K}$ & 1 \\
\hline & 8. & A & $\mathrm{R}$ & $\mathrm{N}$ & $\mathrm{G}$ & $\mathrm{K}$ & $\mathrm{N} / \mathrm{Y}$ & $\mathrm{N}$ & $\mathrm{T}$ & 1 \\
\hline & 9. & A & $\mathrm{R}$ & $\mathrm{N}$ & A & $\mathrm{K}$ & $\mathrm{N}$ & $\mathrm{N}$ & $\mathrm{K}$ & 1 \\
\hline & 10. & A & $\mathrm{C}$ & $S$ & A & $\mathrm{K}$ & $\mathrm{Y}$ & $\mathrm{N}$ & $\mathrm{T}$ & 2 \\
\hline & 11. & A & $\mathrm{R}$ & $\mathrm{N}$ & A & K & - & - & - & 2 \\
\hline & 12. & A & $\mathrm{R}$ & $\mathrm{N}$ & $\mathrm{G}$ & $\mathrm{K}$ & - & - & - & 1 \\
\hline & 13. & A & $\mathrm{C} / \mathrm{R}$ & $\mathrm{S} / \mathrm{N}$ & A & $\mathrm{K}$ & - & - & - & 1 \\
\hline
\end{tabular}

\footnotetext{
* For definition of abbreviations, see Table 1.
} 


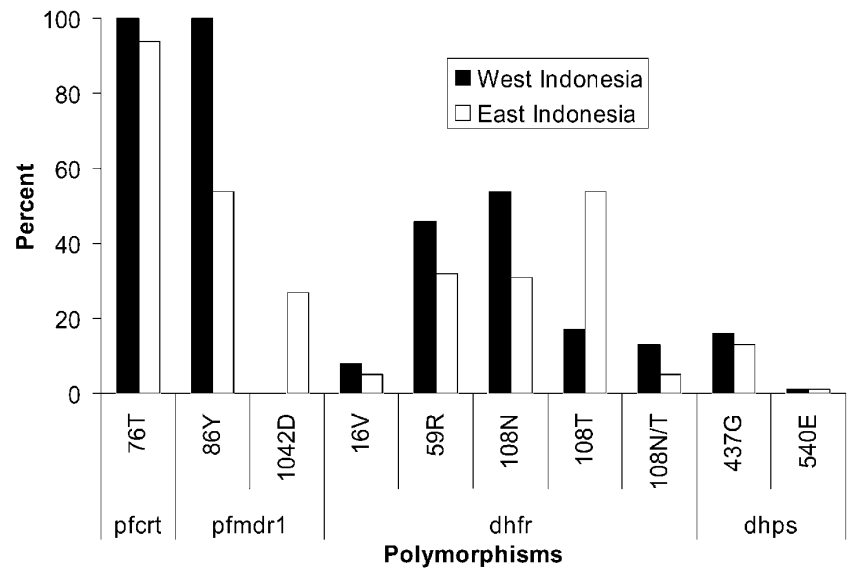

FIGURE 2. Comparison of polymorphisms identified in west and east Indonesia. The 1042D polymorphism of the Plasmodium falciparum multidrug resistance 1 (pfmdr1) gene was only found in eastern Indonesia. pfcrt $=P$. falciparum chloroquine resistance transporter; $\mathrm{dhfr}=$ dihydrofolate reductase; dhps = dihydropteroate synthase.

these were the 1042D polymorphism, without the expected 86Y polymorphism. Recent findings have shown that Papua New Guinea and Papua Indonesia have a range of variants of chloroquine-resistant $P$. falciparum parasites at $p f c r t$ codons 72-76. ${ }^{42,43}$ These variants encompass African, southeast Asian, and South American haplotypes. While work on this area of parasite evolution is still in its early stages, future studies may conceivably show an association between regional pfmdr1 and pfcrt haplotypes. Given that the $p f m d r 1$ $1042 \mathrm{D}$ polymorphism is far more common in South America, further work on this aspect could show interesting results. In isolated Papua, pfcrt 76T appears to be fixed in the parasite population; however, there is only a low rate of polymorphism in $p f m d r 1$. In our previous study in Armopa, Papua Province involving Javanese migrants who have easier access to the Health center than the indigenous Papuans, a high prevalence of $86 \mathrm{Y}$ polymorphism was found in the $P$. falciparum isolates examined. ${ }^{8}$ A closer examination of the social and chemotherapeutic history in this particular area may shed more light on this apparent contradiction. It is of interest to note the occurrence of two isolates in Minahasa carrying both $86 \mathrm{Y}$ and $1042 \mathrm{D}$ polymorphisms of $p f m d r 1$. A recent study also reported this allele from Purworejo district in the island of Java. ${ }^{12}$ This finding may be associated with the civil strife in the eastern parts of Indonesia that has resulted in migration to Java.

Given the spread of chloroquine treatment failure to many parts of Indonesia over the last two decades, $5,7,8,9,11,17,44$ it was not surprising to find that $P$. falciparum isolates in Indonesia carry the polymorphisms in $p f m d r 1$ and $p f c r t$ that have been associated with resistance to this drug.

The $P$. falciparum isolates examined carried polymorphisms in $d h f r$ as either $108 \mathrm{~N}$ or $108 \mathrm{~T}$ as well as the $59 \mathrm{R}$ and $16 \mathrm{~V}$ polymorphisms. There are two antifolate drugs that have been used in Indonesia, pyrimethamine and proguanil (cycloguanil). Pyrimethamine has always been used in combination with sulfadoxine, whereas proguanil is available as a single drug (Paludrine ${ }^{\circledR}$; AstraZeneca Pty., North Ryde, New South Wales, Australia). Evidence to date indicates that the molecular mechanism that underlies resistance to antifolate antimalarials involves the S108N/T polymorphism of $d h f r$. There is a differential target in the dhfr enzyme between pyrimethamine and cycloguanil in which resistance to pyrimethamine is mainly linked to the $108 \mathrm{~N}$ polymorphism, whereas cycloguanil resistance is linked to the $16 \mathrm{~V}$ plus $108 \mathrm{~T}$ polymorphism. However, additional polymorphisms at 50A, 51I, 59R, and 164L will confer cross-resistance to both antifolate drugs. ${ }^{32}$ In our, study there was a relatively common distribution of the 108T $d h f r$ mutation, either as a single polymorphism or else paired with $16 \mathrm{~V}$ among the field isolates of $P$. falciparum from Indonesia. This single polymorphism has been reported in laboratory clones ${ }^{45}$ and enzyme kinetic analysis indicated that this mutant did not confer resistance to cyloguanil. Conversely, the enzyme harboring mutant $16 \mathrm{~V}$ alone showed a high resistance to cycloguanil, but exhibited a very low kinetic parameter, indicating that this mutation may interfere with the fitness of the isolates and that the parasite carrying this polymorphism may not survive in the natural population of the parasite. The findings that no $P$. falciparum isolates in Indonesia carried the $16 \mathrm{~V}$ as a single mutation are in line with this suggestion. However, as this previous work reports, the poor dhfr activity of the A16V polymorphism was restored in the presence of another mutation, 108T. Therefore, it is strongly suspected that the $108 \mathrm{~T}$ mutation in Indonesia may serve as a precursor for the double mutant $16 \mathrm{~V}$ plus $108 \mathrm{~T}$, which is highly resistant to cycloguanil.

The reported distribution of the single $108 \mathrm{~T}$ polymorphism among field isolates of $P$. falciparum has been very limited and so far it has only been seen in isolates from Indonesia and Papua New Guinea. ${ }^{46,47}$ In this regard, its relatively more common distribution among the $P$. falciparum isolates from the eastern part of Indonesia suggests that the isolates carrying this polymorphism may share the same origin with the isolate found in Papua New Guinea.

In this study, polymorphisms in the dhps gene were found less frequently than polymorphisms in other genes. The highest rate of mutation (23\%) was found in northern Sulawesi. The most common mutation was $437 \mathrm{G}$ and in one sample each from Kokap, northern Sulawesi, and Armopa; this was paired with the $540 \mathrm{E}$ polymorphism. The role of dhps polymorphisms in the mechanism of resistance to sulfa drugs has been well described. ${ }^{33,34}$ The presence of the $437 \mathrm{G}$ polymorphism confers resistance to sulfadoxine in $P$. falciparum, and when coupled with the additional polymorphism at $540 \mathrm{E}$ the level of resistance is increased. ${ }^{27}$ Our results indicate that the $P$. falciparum isolates from Indonesia are still predominantly wild-type $d h p s$, a finding that reflects the results of recent studies in the region in Papua New Guinea. ${ }^{48}$

There has been a disturbing report from Nias Island on the high rate of $P$. falciparum treatment failure following sulfadoxine-pyrimethamine chemotherapy. ${ }^{17}$ In this study, no dhps polymorphisms were found in samples from Nias Island, and only very low rates of mutation at the other study sites. Not withstanding the recent encouraging report from Malawi on long-term sulfadoxine-pyrimethamine efficacy, ${ }^{49}$ this is a worrying indicator for Indonesia. The combination of sulfadoxine-pyrimethamine is used as the second-line antimalarial drug in Indonesia. While there is a lack of broad epidemiologic data available, what is published suggests that resistance to this drug combination has spread to malaria-endemic areas in Indonesia. ${ }^{8,15,17,44}$ Given this, serious consideration must now be given to the use of artemisinin combination therapy, 
which has already been shown to be effective against chloroquine-resistant strains of $P$. falciparum in Indonesia. ${ }^{6}$

In conclusion, molecular analysis of $P$. falciparum parasites from eight malaria-endemic areas in Indonesia indicated the widespread presence of isolates carry resistance polymorphisms to both the first-line antimalarial drug chloroquine and to the second-line treatment sulfadoxine-pyrimethamine. With widespread reports of treatment failure and the current increase of malaria morbidity and mortality in Indonesia, serious consideration should be given to the revision of the guidelines for the chemotherapeutic treatment of malaria in Indonesia.

Received January 6, 2004. Accepted for publication July 26, 2004.

Acknowledgments: We thank the officials of the Ministry of Health at the selected areas for providing us with technical support during the malariometric surveys in a the given areas, and Dr. Supargiyono (Gajah Mada University, Yogyakarta, Indonesia) and Dr. P. Hariyanto (Sam Ratulangi University, Manado, Indonesia) for helping us with sample collection. We also thank Professor Sangkot Marzuki (Director of the Eijkman Institute, Jakarta, Indonesia) for his input.

Financial support: This work was supported by the Indonesian and Australian Governments through Bappenas and the Australian Agency for International Development through the Australia Indonesia Medical Research Initiative program. Hadya S. Nagesha was supported by the Wellcome Trust (United Kingdom).

Authors' addresses: Din Syafruddin, Eijkman Institute for Molecular Biology, Jl. Diponegoro 69, Jakarta 10430, Indonesia and Department of Parasitology, Faculty of Medicine, Hasanuddin University, Jl. Perintis Kemerdekaan Km 10, Makassar 90245, Indonesia. Puji B. S. Asih, Eijkman Institute for Molecular Biology, Jl. Diponegoro 69, Jakarta 10430, Indonesia. Gerard J. Casey, Hadya S. Nagesha, and Alan F. Cowman, The Walter and Eliza Hall Institute of Medical Research, Melbourne, Victoria, Australia. Jason Maguire and J. Kevin Baird, United States Naval Medical Research Unit No. 2, Jakarta, Indonesia. John C. Reeder, Papua New Guinea Institute of Medical Research, Goroka EHP441, PO Box 60, Papua New Guinea.

Reprint requests: Din Syafruddin, Eijkman Institute for Molecular Biology, Jalan Diponegoro 69, Jakarta 10430, Indonesia, Telephone: 62-21-3917131, Fax: +62-21-3147982, E-mail: din@eijkman.go.id.

\section{REFERENCES}

1. Ebisawa I, Fukuyama T, 1975. Chloroquine-resistant falciparum malaria from west Irian and east Kalimantan. Ann Trop Med Parasitol 69: 275-282.

2. Clyde DF, McCarthy VC, Miller RM, Hornick RB, 1976. Chloroquine-resistant falciparum malaria from Irian Jaya (Indonesian New Guinea). J Trop Med Hyg 79: 38-41.

3. Pribadi W, 1992. In vitro sensitivity of Plasmodium falciparum to chloroquine and other antimalarials in east Timor and east Kalimantan, Indonesia. Southeast Asian J Trop Med Public Health 23 (Suppl 4): 143-148.

4. Tjitra E, Gunawan S, Laihad F, HM, Sulaksono S, Arjoso S, Richie TL, Manurung N, 1997. Evaluation of antimalarial drugs in Indonesia 1981-1995. Buletin Penelitian Kesehatan (Indonesian) 25: 27-58.

5. Fryauff DJ, Baird JK, Candradikusuma D, Masbar S, Sutamihardja MA, Leksana B, Tuti S, Marwoto H, Richie TL, Romzan A, 1997. Survey of in vivo sensitivity to chloroquine by Plasmodium falciparum and $P$. vivax in Lombok, Indonesia. Am J Trop Med Hyg 56: 241-244.

6. Tjitra E, Suprianto S, Currie BJ, Morris PS, Saunders JR, Anstey NM, 2001. Therapy of uncomplicated falciparum malaria: a randomized trial comparing artesunate plus sulfadoxinepyrimethamine versus sulfadoxine-pyrimethamine alone in Irian Jaya, Indonesia. Am J Trop Med Hyg 65: 309-317.

7. Maguire JD, Susanti AI, Krisin, Sismadi P, Fryauff DJ, Baird JK,
2001. The T76 mutation in the pfcrt gene of Plasmodium falciparum and clinical chloroquine resistance phenotypes in Papua, Indonesia. Ann Trop Med Parasitol 95: 559-572.

8. Nagesha HS, Din S, Casey GJ, Susanti AI, Fryauff DJ, Reeder JC, Cowman AF, 2001. Mutations in the pfmdrl, dhfr and dhps genes of Plasmodium falciparum are associated with in-vivo drug resistance in west Papua, Indonesia. Trans $R$ Soc Trop Med Hyg 95: 43-49.

9. Baird JK, Wiady I, Sutanihardja A, Suradi, Purnomo, Basri H, Sekartuti, Ayomi E, Fryauff DJ, Hoffman SL, 2002. Short report: therapeutic efficacy of chloroquine combined with primaquine against Plasmodium falciparum in northeastern Papua, Indonesia. Am J Trop Med Hyg 66: 659-660.

10. Maguire JD, Lacy MD, Sururi, Sismadi P, Krisin, Wiady I, Laksana B, Bangs MJ, Masbar S, Susanti I, Basuki W, Barcus MJ, Marwoto H, Edstein MD, Tjokrosonto S, Baird JK, 2002. Chloroquine or sulfadoxine-pyrimethamine for the treatment of uncomplicated, Plasmodium falciparum malaria during an epidemic in central Java, Indonesia. Ann Trop Med Parasitol 96: 655-668.

11. Sumawinata IW, Bernadeta, Leksana B, Sutamihardja A, Purnomo, Subianto B, Sekartuti, Fryauff DJ, Baird JK, 2003. Very high risk of therapeutic failure with chloroquine for uncomplicated Plasmodium falciparum and $P$. vivax malaria in Indonesian Papua. Am J Trop Med Hyg 68: 416-420.

12. Syafruddin D, Asih PBS, Aggarwal SL, Shankar AH, 2003. Frequency distribution of antimalarial drug-resistant alleles among isolates of Plasmodium falciparum in Purworejo district, Central Java Province, Indonesia. Am J Trop Med Hyg 69: 614-620.

13. Chulay JD, Watkins WM, Sixsmith DG, 1984. Synergistic antimalarial activity of pyrimethamine and sulfadoxine against Plasmodium falciparum in vitro. Am J Trop Med Hyg 33: 325330.

14. Sirawaraporn W, Yuthavong Y, 1986. Potentiating effect of pyrimethamine and sulfadoxine against dihydrofolate reductase from pyrimethamine-sensitive and pyrimethamine-resistant Plasmodium chabaudi. Antimicrob Agents Chemother 29: 899905.

15. Hoffman SL, Rustama D, Dimpudus AJ, Punjabi NH, Campbell JR, Oetomo HS, Marwoto HA, Harun S, Sukri N, Heizmann $\mathrm{P}, 1985$. RII and RIII type resistance of Plasmodium falciparum to combination of mefloquine and sulfadoxine/ pyrimethamine in Indonesia. Lancet 2: 1038-1040.

16. Baird JK, Basri H, Jones TR, Purnomo, Bangs MJ, Ritonga A, 1991. Resistance to antimalarials by Plasmodium falciparum in Arso PIR, Irian Jaya, Indonesia. Am J Trop Med Hyg 44: 640-644.

17. Fryauff DJ, Leksana B, Masbar S, Wiady I, Sismadi P, Susanti AI, Nagesha HS, Syafruddin D, Atmosoedjono S, Bangs MJ, Baird $\mathrm{JK}, 2002$. The drug sensitivity and transmission dynamics of human malaria on Nias Island, north Sumatra, Indonesia. Ann Trop Med Parasitol 96: 447-462.

18. Bustos DG, Canfield CJ, Canete-Miguel E, Hutchinson DB, 1999. Atovaquone-proguanil compared with chloroquine and chloroquine-sulfadoxine-pyrimethamine for treatment of acute Plasmodium falciparum malaria in the Philippines. $J$ Infect Dis 179: 1587-1590.

19. Cox-Singh J, Zakaria R, Abdullah MS, Rahman HA, Nagappan S, Singh B, 2001. Short report: differences in dihydrofolate reductase but not dihydropteroate synthase alleles in Plasmodium falciparum isolates from geographically distinct areas in Malaysia. Am J Trop Med Hyg 64: 28-31.

20. Wongsrichanalai C, Sirichaisinthop J, Karwacki JJ, Congpuong K, Miller RS, Pang L, Thimasarn K, 2001. Drug resistant malaria on the Thai-Myanmar and Thai-Cambodian borders. Southeast Asian J Trop Med Public Health 32: 41-49.

21. Schwobel B, Jordan S, Vanisaveth V, Phetsouvanh R, Christophel EM, Phompida S, von Sonnenburg F, Jelinek T, 2003. Therapeutic efficacy of chloroquine plus sulphadoxine/ pyrimethamine compared with monotherapy with either chloroquine or sulphadoxine/pyrimethamine in uncomplicated Plasmodium falciparum malaria in Laos. Trop Med Int Health 8: 19-24.

22. Mohapatra PK, Namchoom NS, Prakash A, Bhattacharya DR, 
Goswami BK, Mahanta J, 2003. Therapeutic efficacy of antimalarials in Plasmodium falciparum malaria in an IndoMyanmar border area of Arunachal Pradesh. Indian J Med Res 118: 71-76.

23. Fidock DA, Nomura T, Talley AK, Cooper RA, Dzekunov SM, Ferdig MT, Ursos LM, Sidhu AB, Naude B, Deitsch KW, Su XZ, Wootton JC, Roepe PD, Wellems TE, 2000. Mutations in the $P$. falciparum digestive vacuole transmembrane protein PfCRT and evidence for their role in chloroquine resistance. Mol Cell 6: 861-871.

24. Djimde A, Doumbo OK, Cortese JF, Kayentao K, Doumbo S, Diourte Y, Dicko A, Su XZ, Nomura T, Fidock DA, Wellems TE, Plowe CV, 2001. A molecular marker for chloroquineresistant falciparum malaria. $N$ Engl J Med 344: 257-263.

25. Djimde A, Doumbo OK, Steketee RW, Plowe CV, 2001b. Application of a molecular marker for surveillance of chloroquine-resistant falciparum malaria. Lancet 358: 890-891.

26. Sidhu AB, Verdier-Pinard D, Fidock DA, 2002. Chloroquine resistance in Plasmodium falciparum malaria parasites conferred by $p$ fcrt mutations. Science 298: 210-213.

27. Foote SJ, Thompson JK, Cowman AF, Kemp DJ, 1989. Amplification of the multidrug resistance gene in some chloroquineresistant isolates of $P$. falciparum. Cell 57: 921-930.

28. Reed MB, Saliba KJ, Caruana SR, Kirk K, Cowman AF, 2000. Pgh1 modulates sensitivity and resistance to multiple antimalarials in Plasmodium falciparum. Nature 403: 906-909.

29. Cowman AF, Morry MJ, Biggs BA, Cross GA, Foote SJ, 1988. Amino acid changes linked to pyrimethamine resistance in the dihydrofolate reductase-thymidylate synthase gene of Plasmodium falciparum. Proc Natl Acad Sci U S A 85: 9109-9113.

30. Peterson DS, Walliker D, Wellems TE, 1988. Evidence that a point mutation in dihydrofolate reductase-thymidylate synthase confers resistance to pyrimethamine in falciparum malaria. Proc Natl Acad Sci U S A 85: 9114-9118.

31. Foote SJ, Galatis D, Cowman AF, 1990. Amino acids in the dihydrofolate reductase-thymidylate synthase gene of Plasmodium falciparum involved in cycloguanil resistance differ from those involved in pyrimethamine resistance. Proc Natl Acad Sci U S A 87: 3014-3017.

32. Peterson DS, Milhous WK, Wellems TE, 1990. Molecular basis of differential resistance to cycloguanil and pyrimethamine in Plasmodium falciparum malaria. Proc Natl Acad Sci U S A 87: 3018-3022.

33. Triglia T, Wang P, Sims PF, Hyde JE, Cowman AF, 1998. Allelic exchange at the endogenous genomic locus in Plasmodium falciparum proves the role of dihydropteroate synthase in sulfadoxine-resistant malaria. EMBO J 17: 3807-3815.

34. Triglia T, Cowman AF, 1999. The mechanism of resistance to sulfa drugs in Plasmodium falciparum. Drug Resist Update 2: $15-19$.

35. Plowe CV, Cortese JF, Djimde A, Nwanyanwu OC, Watkins WM, Winstanley PA, Estrada-Franco JG, Mollinedo RE, Avila JC, Cespedes JL, Carter D, Doumbo OK, 1997. Mutations in Plasmodium falciparum dihydrofolate reductase and dihydropteroate synthase and epidemiologic patterns of pyrimethamine- sulfadoxine use and resistance. J Infect Dis 176: $1590-1596$.

36. Vasconcelos KF, Plowe CV, Fontes CJ, Kyle D, Wirth DF, Pereira da Silva LH, Zalis MG, 2000. Mutations in Plasmodium falciparum dihydrofolate reductase and dihydropteroate synthase of isolates from the Amazon region of Brazil. Mem Inst Oswaldo Cruz 95: 721-728.

37. Basco LK, Ndounga M, Ngane VF, Soula G, 2002. Molecular epidemiology of malaria in Cameroon. XIV. Plasmodium falciparum chloroquine resistance transporter (PFCRT) gene sequences of isolates before and after chloroquine treatment. Am J Trop Med Hyg 67: 392-395.

38. Wooden J, Kyes S, Sibley CH, 1993. PCR and strain identification in Plasmodium falciparum. Parasitol Today 9: 303-305.

39. Duraisingh MT, Drakeley CJ, Muller O, Bailey R, Snounou G, Targett GA, Greenwood BM, Warhurst DC, 1997. Evidence for selection for the tyrosine-86 allele of the $p f m d r 1$ gene of Plasmodium falciparum by chloroquine and amodiaquine. Parasitology 114: 205-211.

40. Duraisingh MT, Curtis J, Warhurst DC, 1998. Plasmodium falciparum: detection of polymorphisms in the dihydrofolate reductase and dihydropteroate synthetase genes by PCR and restriction digestion. Exp Parasitol 89: 1-8.

41. Gomez-Saladin E, Fryauff D, Taylor W, Laksana B, Susanti A, Purnomo, Subianto B, Richie T, 1999. Plasmodium falciparum mdr1 mutations and in vivo chloroquine resistance in Indonesia. Am J Trop Med Hyg 61: 240-244.

42. Nagesha HS, Casey GJ, Riekmann KH, Fryauff DJ, Laksana BS, Reeder JC, Maguire JD, Baird JK, 2003. New haplotypes of the Plasmodium falciparum chloroquine resistance transporter ( $p f c r t)$ gene among chloroquine-resistant parasite isolates. Am J Trop Med Hyg 68: 398-402.

43. Mehlotra RK, Fujioka H, Roepe PD, Janneh O, Ursos LM, Jacobs-Lorena V, McNamara DT, Bockarie MJ, Kazura JW, Kyle DE, Fidock DA, Zimmerman PA, 2001. Evolution of a unique Plasmodium falciparum chloroquine-resistance phenotype in association with pfcrt polymorphism in Papua New Guinea and South America. Proc Natl Acad Sci U S A 98: 12689-12694.

44. Fryauff DJ, Sumawinata I, Purnomo, Richie TL, Tjitra E, Bangs MJ, Kadir A, Ingkokusumo G, 1999. In vivo responses to antimalarials by Plasmodium falciparum and Plasmodium vivax from isolated Gag Island off northwest Irian Jaya, Indonesia. Am J Trop Med Hyg 60: 542-546.

45. Sirawaraporn W, Sathitkul T, Sirawaraporn R, Yuthavong Y, Santi DV, 1997. Antifolate-resistant mutants of Plasmodium falciparum dihydrofolate reductase. Proc Natl Acad Sci U S A 94: 1124-1129.

46. Reeder JC, Rieckmann KH, Genton B, Lorry K, Wines B, Cowman AF, 1996. Point mutations in the dihydrofolate reductase and dihydropteroate synthetase genes and in vitro susceptibility to pyrimethamine and cycloguanil of Plasmodium falciparum isolates from Papua New Guinea. Am J Trop Med Hyg 55: 209-213.

47. Syafruddin D, Asih PB, Siregar JE, Tjitra E, 2003. Molecular basis of antimalarial drug resistance in Indonesia. $A d v$ Exp Med Biol 531: 103-115.

48. Casey GJ, Ginny M, Uranoli M, Mueller I, Reeder JC, Genton B, Cowman AF, 2004. Molecular analysis of Plasmodium falciparum from drug treatment failure patients in Papua New Guinea. Am J Trop Med Hyg 70: 251-255.

49. Plowe CV, Kublin JG, Dzinjalamala FK, Kamwendo DS, Mukadam RAG, Chimpeni P, Molyneux ME, Taylor TE, 2004. Sustained clinical efficacy of sulfadoxine-pyrimethamine for uncomplicated falciparum malaria in Malawi after 10 years as first line treatment: five year prospective study. BMJ 328: 545 . 